\title{
Reaction between Chlorine Extreme Pressure Additives and Metal Surfaces at High Temperatures*
}

\author{
Toshio Sakurai**, Kachio Sato*** and Yoshio Yamamoto****
}

\begin{abstract}
Summary: The high temperature corrosion of iron was investigated in hydrocarbon oils containing chlorine type EP additives, using the hot wire method ${ }^{1}$.

With regard to the corrosion rate, both the parabolic law and the cubic law were observed. The former law was demonstrated by the diffusion mechanism of corrosion. The cubic law was shown by the retarding action of a barrier film which was formed on the reacting surfaces, causing the decomposition of the chlorine compounds.

The complexity of the chlorine corrosion mechanism was revealed by using $X$-ray analysis on the formed films. The axidation reaction and hydrolysis of formed iron chloride had to be taken into consideration in conjunction with the chlorination reaction.

In the correlation between chemical reactivity and load carrying capacity of oils containing chlorine compounds, it has been found that more active oils exhibit higher load carrying capacity.

Sulfur compounds were observed to yield higher Mean Hertz Load values than chlorine compounds of the same chemical reactivity. Conceivably, the solid lubricant films formed by sulfur and oxygen possess higher load carrying capacity than those formed by chlorine.
\end{abstract}

\section{Introduction}

In the modern theory of lubrication, it is generally accepted that the anti-wear and anti-friction properties of EP additives are connected with their chemical reactivity toward metals ${ }^{2}$. Under boundary lubrication conditions of local high temperature and pressure on the rubbing surfaces, EP agents are believed to chemically attack the metal surfaces to from a solid film which acts as a boundary lubricant. This chemical attack, or reaction, appears to be so significant as to warrant an investigation of the high temperature reaction of EP agents and metal surfaces in order to provide a further understanding of EP lubrication.

We previously reported about the reaction between sulfur compounds and metal surfaces

* Received December 7, 1964.

** Tokyo Institute of Technology, 1 Ookayama, Meguroku, Tokyo.

*** Central Research Laboratory, Nippon Mining Co. Ltd., Todamachi, Kita-Adachi-gun, Saitama Pref.

**** Tokyo Institute of Technology, present address Daikyo Oil Co. Ltd., Research Laboratory Yokkaichi. at high temperatures, using the hot wire method $^{33}$.

Some interesting considerations are given for both chlorine compounds and sulfur compounds concerning the mechanism of corrosion and the correlation between chemical reactivity and load carrying capacity.

\section{Experimental}

Apparatus and Experimental Procedure

The apparatus used was the same as reported in the previous paper ${ }^{3}$. The apparatus was designed to provide a measuring and a heating circuit.

A thin iron wire supported across two terminals was immersed in a sample oil and the electrical resistance of the wire was measured by the measuring circuit. Once connected to the heating circuit, the wire was heated electrically, and was corroded. The reaction-temperature was deduced from the resistance temperature characteristics of the iron used. After the reaction, the change of the electrical resistance was measured.

Assuming that the reaction products formed 
on the wire surfaces are non-conducting and the attack by EP agents is uniform over the whole of the metal surfaces, the "change in resistance" may be converted to the "thickness of iron corroded" by the following equation:

$$
J r \approx\left(\frac{r_{0}}{2}\right)\left(\frac{\Delta R}{R_{0}+\Delta R}\right)
$$

where $J r=$ thickness of iron corroded, $\Delta R=$ change in resistance due to corrosion, $R_{0}=$ initial resistance of wire, and $r_{0}=$ radius of wire used.

Since the thickness of iron corroded is easily deduced from the change in electrical resistance, the amounts of corrosion may be successfully obtained in various solutions. The corrosion process was carried out in various chlorine compound solutions. X-ray analysis of the reaction products was conducted in order to obtain further knowledge concerning the corrosion of iron.

Mean Hertz Load values for the oils containing chlorine EP agents were determined in conformity with Federal Test Method 6503. A correlation between the chemical reactivity and load carrying capacity of these materials was established.

\section{Materials}

Chlorine compounds used were monochlorobenzene (b. p. $130-130.5^{\circ} \mathrm{C}$ ), benzylchloride (b. p. $109-110^{\circ} \mathrm{C} / 10 \mathrm{mmHg}$ ), hexachloroethane (m. p. $\left.183-185^{\circ} \mathrm{C}\right)$, chlorinated paraffin (chlorine content, 41.0 wt \%), methyltrichlorostearate (chlorine content, $22.7 \mathrm{wt} \%$ ), methylpentachlorostearate (chlorine content, 32.4 wt\%) and pentachlorodiphenyl. Highly purified white oil was used as a solvent.

The thin iron wire used was a piano wire, $(0.04 \mathrm{~mm}$ in diameter). Prior to the experiments, the wire was vacuum-annealed for two hours under conditions of $600^{\circ} \mathrm{C}$ and $10^{-4} \mathrm{mmHg}$, to ensure accurate results during testing.

\section{Experimental Results}

Corrosion of Iron by Chlorine Compounds

The corrosion process of iron was conducted at various reaction temperatures. Fig. 1 shows the experimental results obtained for white oil alone (without additives) and white

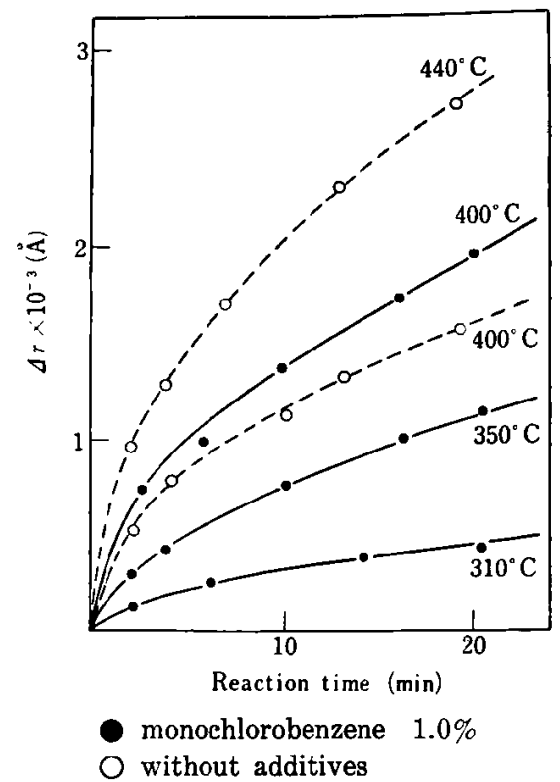

Fig. 1 Corrosion of iron in white oil containing chlorine compounds

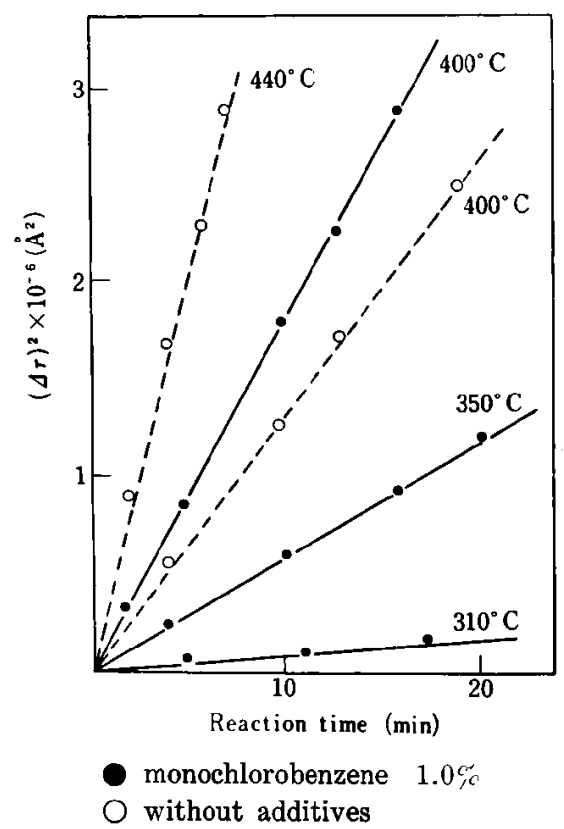

Fig. 2 Relation between the square of thickness of iron corroded and reaction time

oil containing monochlorobenzene. The thickness of iron corroded is shown to generally increase as the reaction temperature is raised. Fig. 2 presents the relation between the square of thickness of iron corroded and the reaction time. As shown in Fig. 2, there is a linear relation between these two factors. Accordingly, the corrosion rate can be expressed by the equation $(\Delta \mathbf{r})^{2}=k_{2} t$, where $\Delta r=$ thickness of iron corroded, $t=$ 


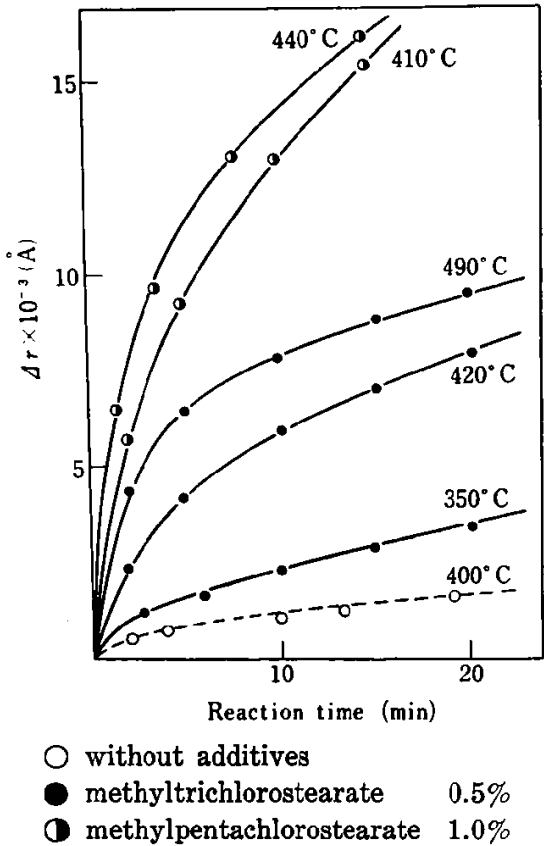

Fig. 3 Corrosion of iron in white oil containing chlorine compounds

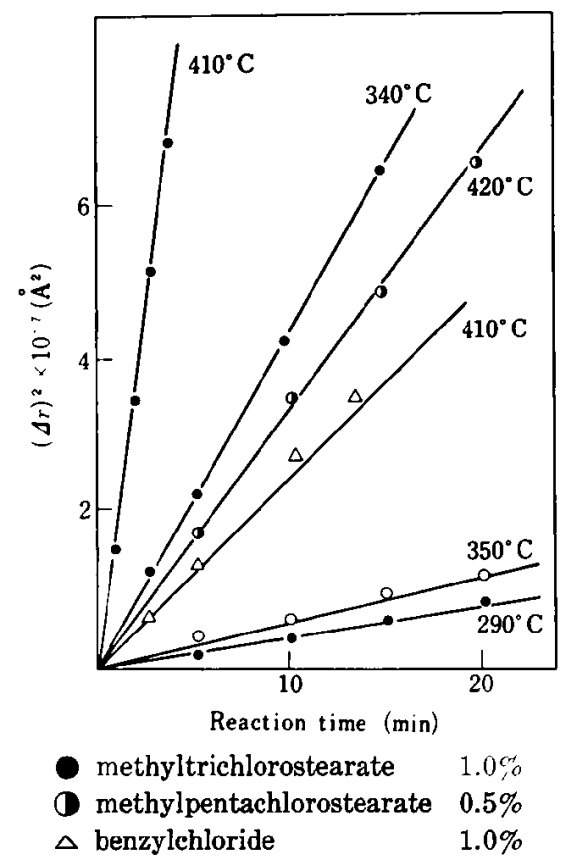

Fig. 4 Relation between the square of thickness of iron corroded and reaction time

reaction time, and $k_{2}=$ corrosion rate constant.

Experimental results for the corrosion process involving methyltrichlorostearate and methylpentachlorostearate are given in Fig. 3. Fig. 4 shows the relation between the square of thickness of iron corroded and the reaction time. Fig. 5 presents the relation between the cube of thickness of iron corroded and reacction time. As shown in Fig. 4 and

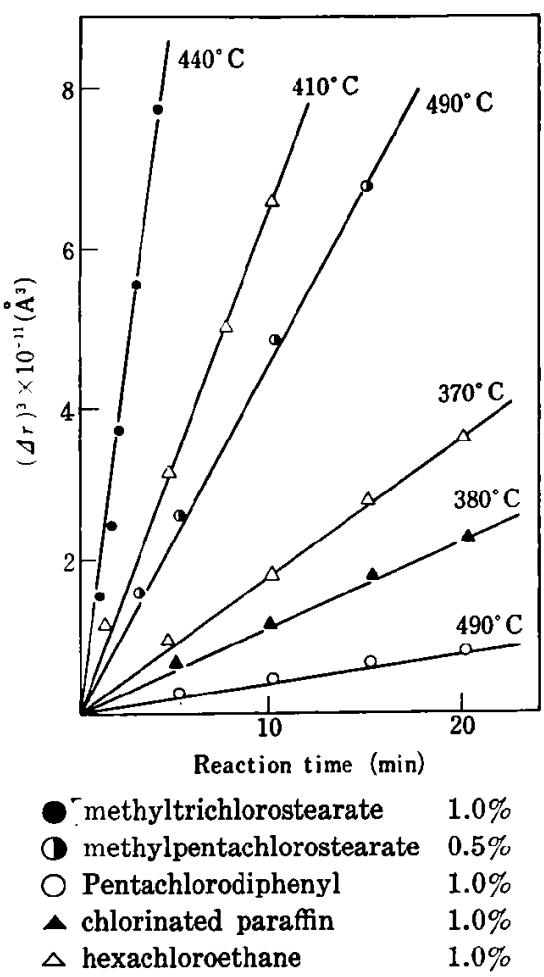

Fig. 5 Relation between the cube of thickness of iron corroded and reaction time

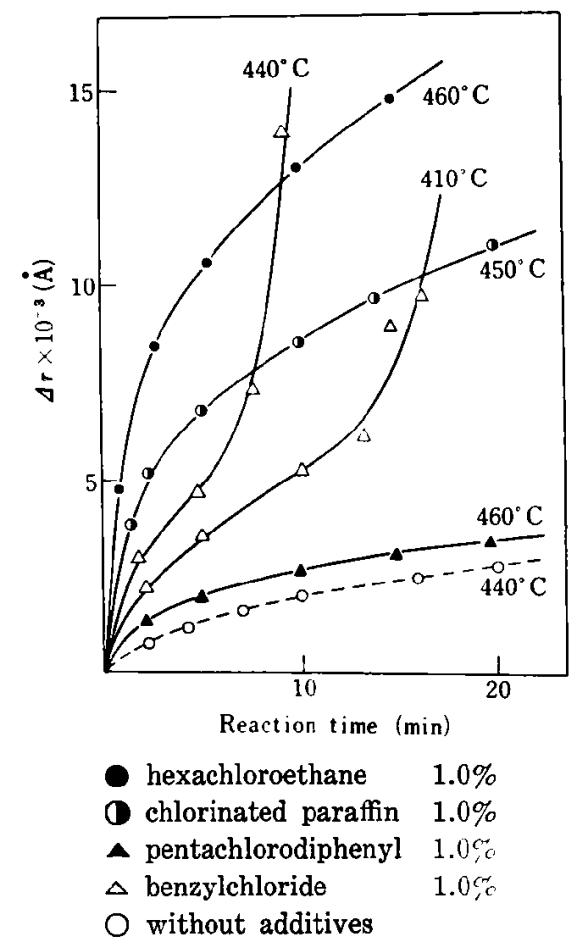

Fig. 6 Corrosion of iron in white oil solutions of chlorine compounds

Fig. 5, the corrosion rate can be given by the equation $(\Delta r)^{2}=k_{2} t$ at lower reaction temperatures, and equation $(\Delta r)^{3}=k_{3} t$ at higher temperatures.

Experimental results for the corrosion 


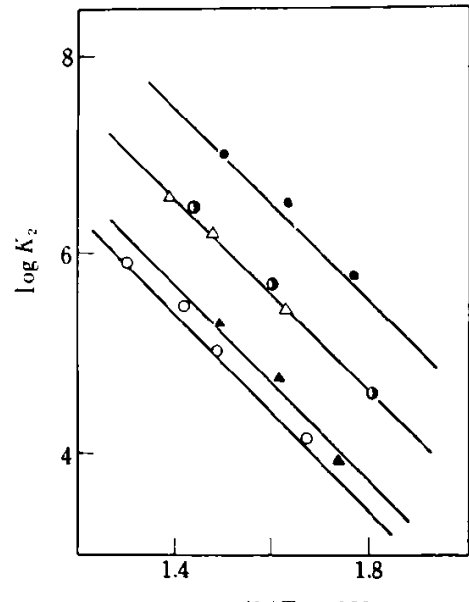

(1) $T) \times 10^{3}$

- methyltrichlorostearate $1.0, c$ (1) methylpentachlorostearate 0.5

$\triangle$ benzylchloride $1.0 \%$

A monochlorobenzene $\quad 1.0$

$O$ without additives

Fig. 7 Arrhenius Plot of $K_{:}$

processes using hexachloroethane, chlorinated paraffin, pentachlorodiphenyl and benzyl chloride, respectively, are presented in Fig. 6. The equation for corrosion rate based on the results obtained for solutions of hexachloroethane, chlorinated paraffin, and pentachlorodiphenyl is seen to be $(\Delta r)^{3}=k_{3} t$. In the case of benzyl chloride solutions, the corrosion rate seems to follow the parabolic law at the initial stage, but a remarkable increase of corrosion and deviation from the parabolic law are observed after the initial

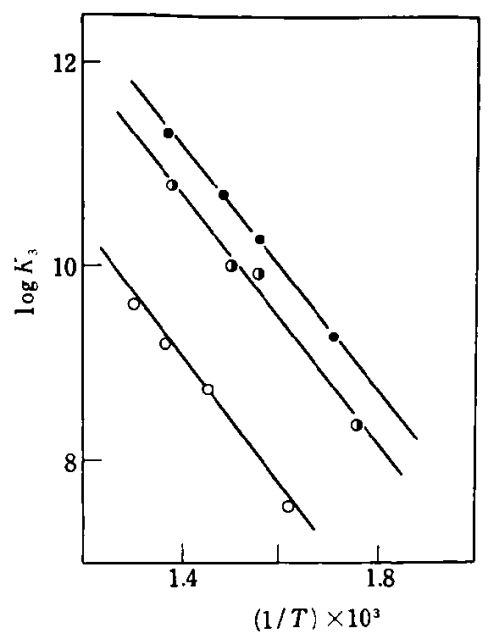

hexachlorothane $1.0 \%$

o chlorinated paraffin $1.0 \%$

pentachlorodiphenyl $1.0 \%$

Fig. 8 Arrhenius plot of $K_{3}$

stage of corrosion.

The data were fitted to the Arrhenius equation, and the calculated value for apparent activation energy was $20 \mathrm{kcal} / \mathrm{mole}$ with $k_{2}$ and $30 \mathrm{kcal} / \mathrm{mole}$ with $k_{3}$ (from Fig. 7 and Fig. 8, respectively).

\section{$X$-ray Analysis of Reaction Products}

The results of $\mathrm{X}$-ray analysis are shown in Fig. 9. $\mathrm{Fe}_{3} \mathrm{O}_{4}$ was identified on the iron surfaces corroded in white oil containing no additives. When monochlorobenzene or pentachlorodiphenyl was used as an additive, the formation of $\mathrm{Fe}_{3} \mathrm{O}_{4}$ was observed, but iron chloride could not be detected. The forma-

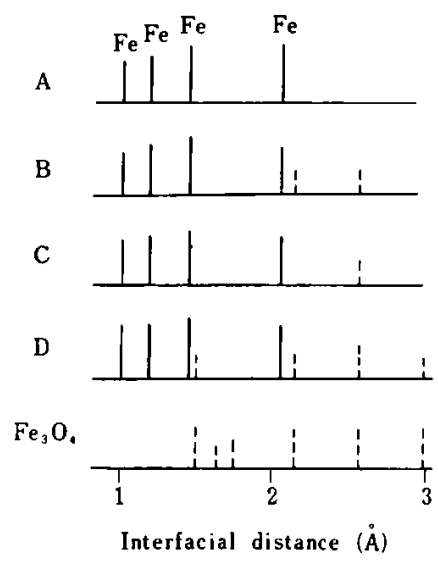

A : iron wire used

B : without additives, $490^{\circ} \mathrm{C}$

$\mathrm{C}$ : monochlorobenzene, $1.0 \%, 400^{\circ} \mathrm{C}$

$\mathrm{D}$ : pentachlorodiphenyl, 1.0$)^{\prime \prime}, 490^{\circ} \mathrm{C}$
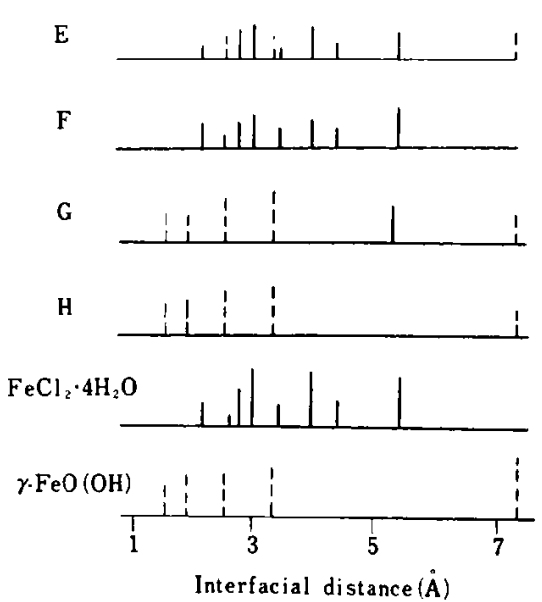

$\mathrm{E}$ : methyltrichlorostearate, $1.0 \%, 410^{\circ} \mathrm{C}$

F : methylpentachlorostearate, $0.5 \%, 490^{\circ} \mathrm{C}$

$\mathrm{G}$ : hexachloroethane, $1.0 \%, 460^{\circ} \mathrm{C}$

$\mathrm{H}$ : chlorinated paraffin, $1.0 \%, 470^{\circ} \mathrm{C}$

Fig. $9 \mathrm{X}$-ray analysis of formed films 
tion of $\mathrm{FeCl}_{2} \cdot 4 \mathrm{H}_{2} \mathrm{O}$ or $\gamma-\mathrm{FeO}(\mathrm{OH})$ was observed when methyltrichlorostearate, methylpentachlorostearate, hexachloroethane or chlorinated paraffin were used as additives.

The Determination of Mean Hertz Load

In order to investigate the correlation between reactivity and load carrying capacity, the Mean Hertz Load of oils containing chlorine or sulfur EP addivives was measured according to the procedure described in $\mathrm{Fe}$ deral Test Method 6503. The experimental results are presented in Table 1.

Table 1 Mean Hertz Load values and corrosion rate constants calculated from Fig. 7 or Fig. 8

\begin{tabular}{lr|r|r}
\hline \multicolumn{1}{c}{ Sample oil } & \multicolumn{2}{c}{$\begin{array}{c}\text { Mean } \\
\text { Hertz Load }\end{array}$} & Log $K^{*}$ \\
\hline Base oil & & 20.98 & 5.07 \\
Monochlorobenzene & $1.0 \%$ & 21.82 & 5.22 \\
Pentachlorodiphenyl & $1.0 \%$ & 22.35 & 8.42 \\
Methylpentachlorostearate & $0.5 \%$ & 28.37 & 6.20 \\
Chlorinated paraffin & $1.0 \%$ & 30.86 & 10.11 \\
Hexachloroethane & $1.0 \%$ & 31.10 & 10.51 \\
Methyltrichlorostearate & $1.0 \%$ & 33.93 & 7.05 \\
Benzylchloride & $1.0 \%$ & 42.28 & 6.20 \\
Dibenzyldisulfide** & $0.5 \%$ & 44.80 & 5.11 \\
Elementary sulfur & $0.5 \%$ & 68.23 & 5.55 \\
\hline * Log $K$ values were calculated at & $400^{\circ} \mathrm{C}$ & \\
** Reference (3) & \multicolumn{2}{c}{}
\end{tabular}

\section{Discussion}

Chemical Reaction in the Process of Corrosion

Even when the white oil was employed alone, considerable corrosion of iron was found to occur on the wire surfaces, and $\mathrm{Fe}_{3} \mathrm{O}_{4}$ was detected there by means of X-ray analysis.

With both monochlorobenzene and pentachlorodiphenyl respectively, it has been found that a similar amount of corrosion takes place to that obtained with white oil without additives; and $\mathrm{Fe}_{3} \mathrm{O}_{4}$ is similarly formed as a reaction product. On the other hand, methyltrichlorostearate and methylpentachlorostearate show strong corrosiveness, and yield iron chloride as a reaction product.

Hence, it should be considered that both the oxidation reaction and the chlorination reaction of iron might take place simultaneously because of the coexistence of dissolved oxygen and chlorine compounds in the solution during the process of corrosion.

It is diffiicult to discuss precisely here which reaction predominates during corrosion. From the data obtained by X-ray analysis, however, it appears that chemical reactivity of chlorine compounds may play a significant role in determining the chemical reactions involved in corrosion. The oxidation reaction might be predominant in the case of corrosion by monochlorobenzene or pentachlorodiphenyl. These exhibit less chemical reactivity toward iron. On the contrary, the chlorination reaction would become predominant when an iron surface was allowed to corrode in a solution of methyltrichlorostearate or methylpentachlorostearate. These have higher reactivity. Furthermore, the iron chloride may be so unstable that hydrolyzed products are formed. In fact, $\gamma-\mathrm{FeO}(\mathrm{OH})$ was detected on the iron surfaces corroded by hexachloroethane and chlorinated paraffin.

Because of the subsequent hydrolysis of the chlorination or oxidation products, it is accepted that chlorine corrosion reactions on iron surfaces are very complicated.

This technique, therefore, makes no distinction between the above reactions, but merely measures each reaction product as a similar non-conducting substance.

\section{Reaction Kinetics of Corrosion}

The mechanism of the corrosion process investigated here may be divided into three groups, as shown schematically in Fig. 10.

With the first group (which is composed of the white oil alone, monochlorobenzene and methyltrichlorostearate), the parabolic law is shown to apply at lower reaction temperatures.

In the second group (represented by hexachloroethane and chlorinated paraffin), the cubic law applies.

An exceptional instance is observed to occur in the corrosion of benzyl chloride. The reaction rate seems to follow the parabolic law at the intitial stage of corrosion, but after that, there occurs a remarkable increase of corrosion and marked deviation from the parabolic law.

As reported in a previous paper, the corrosion reaction being investigated here is conceivably a kind of tarnishing or film forming reaction, whose reaction products become a barrier for further corrosion ${ }^{32}$. Extensive 

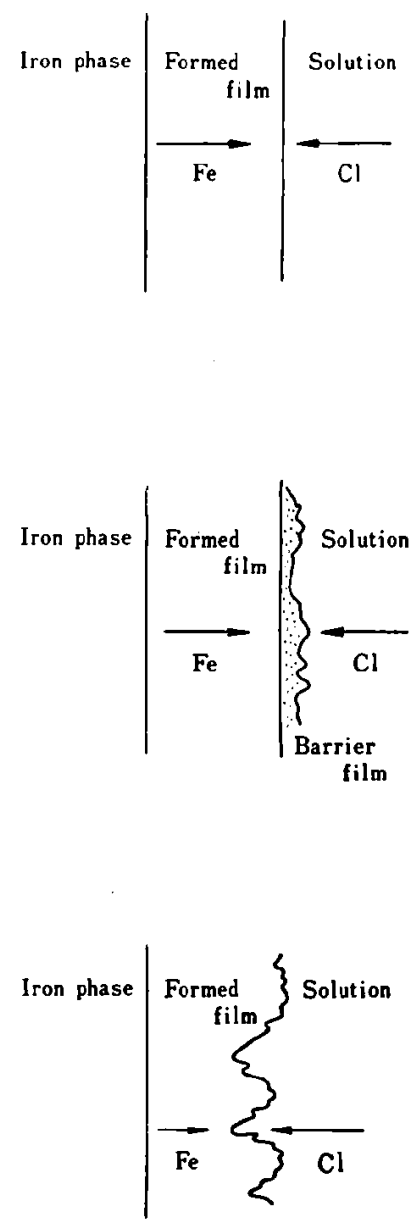

Parabolic law: $(\Delta r)^{2}=K_{2} t$

can be understood by the diffusion mechanism

of corrosion.
Cubic law : $(\Delta r)^{s}=K_{3} t$

may be explained by retarding action of

another barrier films.

Remarkable increase of corrosion will be

caused by the occurence of crack or cleavage.

Fig. 10 Corrosion mechanism of irom

basic studies on tarnishing reaction of metals in the atmosphere have been conducted at high temperatures ${ }^{4,5}$.

If the crust formed on the metal is porous, it will crack; and new metal surfaces will be constantly exposed. In this case the film growth is linear with time. However, the film formed may in general become a barrier to further growth; in such a case, the reaction will slow down and eventually stifle itself. Further film growth is determined by the diffusion of iron, and the corrosion rate follows the parabolic law.

The diffusion mechanism of corrosion may be successfully applicable to the first group as observed in sulfur corrosion ${ }^{3}$. When benzylchloride was used as an additive, there was a remarkable increase of corrosion. This phenomenon might be explained by the occurance of crack or cleavage, which results in a decrease of the formed film's reaction resistivity. After cracking, however, it is doubtful that the amount of corrosion can be measured precisely, because the electrical resistance should enormously increase at the area locally attacked.

The mechanism to explain the cubic law may be proposed as follows:

If the diffusion of iron ion vacancy ( $\mathrm{Fe} \square$ ') is assumed to be a rate determining step, the corrosion rate should be inversely proportional to the thickness of iron corroded and directly proportional to the concentration of $\mathrm{Fe} \square$ " on the iron surfaces. Hence, the corrosion rate is given by the following equation:

$$
\frac{d(\Delta r)}{d t}=\frac{k_{1} C}{J r}
$$

where $\Delta r=$ thickness of iron corroded, $t=$ reaction time, $C=$ concentration of $\mathrm{Fe} \square$ ' on the iron surfaces and $k_{1}=$ constant. By integrating the above equation, the parabolic law is derived.

The reaction resistivity depends only on the formed film on the iron surfaces in the parabolic law. In the cubic law, however, 
another barrier film, caused by the polymerization or carbonization of chlorine compounds must be taken into consideration. This barrier film which deposits on the surface exhibits a retarding action to the surface reaction, and decreases the surface concentration of iron ion vacancy. The schematic explanation for these corrosion mechanisms is shown in Fig. 10.

The barrier film may grow as the corrosion proceeds, and its retarding action will become more significant. Hence, the following equation may be assumed, since the surface concentration of iron ion vacancy is more remarkably decreased as the barrier film increases.

$$
C=\frac{k}{\Delta r}
$$

From equations [2] and [3], the following equation is obtained.

$$
\frac{d(\Delta r)}{d t}=\frac{k_{3}}{(\Delta r)^{2}}
$$

Integrating equation [4], the cubic law is derived:

$$
(\lrcorner r)^{3}=k_{3} t
$$

Chemical Reactivity of Chlorine Compounds

As mentioned above, corrosion processes with chlorine compounds are composed of various indistiguishable chemical reactions such as chlorination, hydrolysis of chlorinated products, and oxidation. In this experiment, no knowledge is obtained concerning the rate constant of chlorination by chlorine compounds. However, relative reactivity of chlorine compounds can be deduced from the Arrhenius plot of rate constant of corrosion; and considerable knowledge may be derived concerning the correlation between the chemical reactivity and the chemical structure.

From the experimental results using monochlorobenzene and pentachlorodiphenyl, it may be accepted that the chlorine atom, combined with a benzene ring, shows very poor reactivity, even at high temperatures. The same fact was observed about the corresponding sulfur compounds in a previous paper. Diphenyl disulfide was shown to exhibit very poor reactivity and the corrosion reaction was dependent mainly upon the oxidation of iron by dissolved oxygen. On the contrary, benzylchloride shows strong corrosiveness toward iron, and a corrosion promoting action as well. The same experimental results were observed as with the corrosion in dibenzyldisulfide solutions. Accordingly, a chlorine atom or a sulfur atom when combined with a benzyl group may be concluded to possess more activity toward iron than when the same atom is combined with a phenyl group. The above conclusion is supported by the electronic theory of organic chemistry.

Correlation between Chemical Reactivity and Load Carrying Capacity

The reactivity of various EP lubricants at high temperatures should affect their load carrying capacity. It is, then, presumably postulated that there is good correlation between chemical reactivity and load carrying capacity.

The following parameter may be introduced as a measure for relative reactivity of chlorine compounds:

$$
(\log k)_{\mathrm{EP}} /(\log k)_{\mathrm{Base}} * \text {, }
$$

where $(\log k)_{E \mathrm{P}}=\log k$ at $400^{\circ} \mathrm{C}$ for the white oil solutions containing EP additives and $(\log k)_{\mathrm{Bas} \theta}=\log k$ at $400^{\circ} \mathrm{C}$ for white oil alone.

Similarly, the relative load carrying capacity of oils containing EP additives can be expressed by the following parameter:

$$
\text { (M.H.L.) })_{E P} /(\text { M.H.L. })_{\text {Base }} \text {, }
$$

where (M.H.L.) $)_{\mathrm{EP}}=$ Mean Hertz Load for white oil solutions containing EP additives and (M.H.L. $)_{\mathrm{Base}}=$ Mean Hertz Load for white oil alone.

Fig. 11 represents the correlation between $(\log k)_{\mathrm{EP}} /(\log k)_{\mathrm{Base}}$ and (M.H.L. $)_{\mathrm{EP}} /(\text { M.H. L. })_{\text {Base }}$ for seven chlorine compounds and two sulfur compounds. As shown in Fig. 11, good correlation can be found between chemical reactivity and load carrying capacity. As to the exceptional case of benzyl chloride, its peculiar charactristics in corrosion must be taken into consideration. The rate constant of corrosion with benzylchloride was cal-

* With the chlorine compounds which follow the cubic law,

$$
\left[\left(\log K_{8}\right)_{\mathrm{EP}} /\left(\log K_{2}\right)_{\mathrm{Bzse}}\right] \times\left(\frac{2}{3}\right)
$$

was used as a parameter of chemical reactivity, because the reactivity must be compared on the same base. 


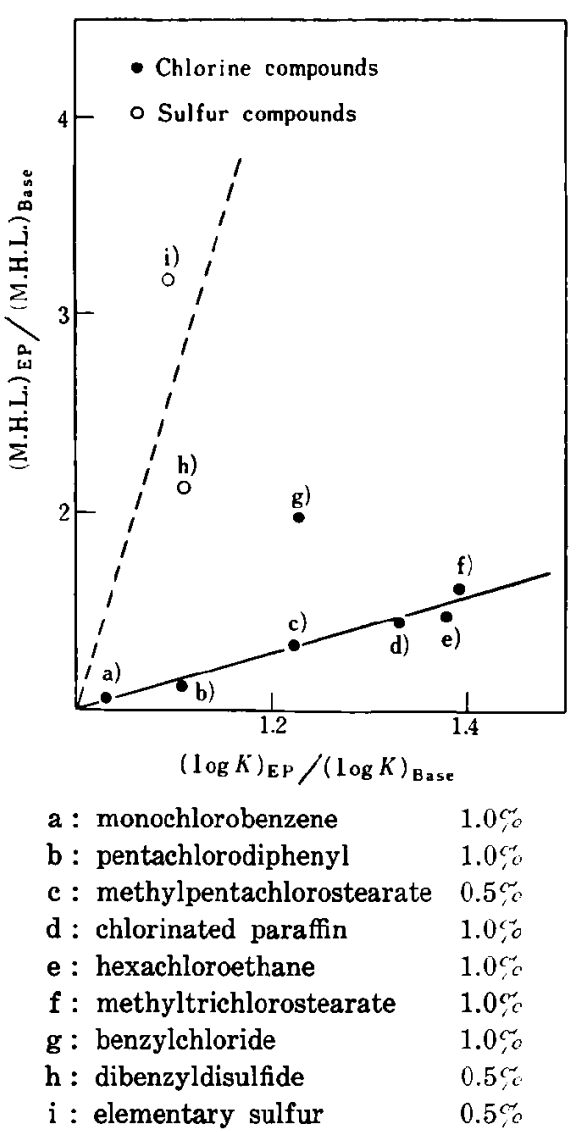

Fig. 11 Correlation between chemical reactivity and load carrying capacity

culated from the results of the initial stage, before the beginning of corrosion promoting action.

It is reasonably assumed from the above correlation that EP additives must possess enough reactivity to form a solid lubricant, in situ, during rubbing, wherever it is required. However, EP additives should only react when operating conditions become severe enough to warrant a corrosive attack. Consequently, over reactive compounds may be inadequate as EP additives.

As shown in Fig. 11, sulfur compounds present more load carrying capacity than the chlorine compounds of the same reactivity. The solid lubricant films formed by sulfur compounds possess higher load carrying capacity than those formed by chlorine compounds, with the materials tested.

\section{Conclusions}

The high temperature corrosion of iron was carried out in hydrocarbon oils con- taining chlorine compounds to develop knowledge of EP lubrication and general chlorine corrosion at high temperatures.

a) It has been observed that the corrosion rate of iron follows the parabolic law or the cubic law. The parabolic law may be understood by the diffusion mechanism of corrosion. In order to explain the cubic law, the retarding action of another barrier film has been assumed.

b) From X-ray analysis of the formed films, the complexity of corrosion processes has been revealed. They are composed of various chemical reactions such as chlorina-, tion, hydrolysis of chlorinated products, and oxidation.

c) The chlorine atom combined with a benzyl group exhibits far stronger reactivity than the chlorine atom combined with a phenyl group. Moreover, benzylchloride has a corrosion promoting action, resulting from the occurrence of a crack on the iron surfaces. Aliphatic chlorine compounds, such as hexachloroethane, chlorinated paraffin or methyl trichlorostearate, exhibit strong chemical reactivity at high temperatures.

d) There seems to be an interesting correlation between chemical reactivity of chlorine compounds and the Mean Hertz Load values of oils containing them. This correlation may help in evaluating EP lubricants practically.

e) Sulfur compounds present higher Mean Hertz Load values than chlorine compounds of the same chemical reactivity. It may be concluded that solid lubricant films formed by sulfur possess higher load carrying capacity than those formed by chlorine.

\section{References}

1) Barcroft, F.T., Wear, 3, 440 (1960).

2) Bowden, F.P., and Tabor, D., The fricrion and lubrication of solid, Oxford University Press, London 33 (1950).

3) Sakurai, T., Sato, K., Ishida, K., Bulletin of the Japan Petroleum Institute, 6, 40 (1964).

4) Miley, H. A., Trans. Electrochem. Soc., 81, 391 (1942).

5) Smeltzer, W.W., Corrosion, 11, 366 (1955). 